\title{
Efeitos da Heterogeneidade de Variância Residual entre Grupos de Contemporâneos na Avaliação Genética de Bovinos de Corte $^{1}$
}

\author{
Roberto Carvalheiro ${ }^{2}$, Luiz Alberto Fries ${ }^{3}$, Flávio Schramm Schenkel ${ }^{4}$, Lucia Galvão de Albuquerque ${ }^{5}$
}

RESUMO - O objetivo deste estudo foi investigar, por meio de dados simulados, o efeito da heterogeneidade de variância residual entre grupos de contemporâneos (GC) sobre as avaliações genéticas de bovinos de corte, e comparar o uso de uma avaliação genética ponderada $\left(\mathrm{R} \neq \mathrm{I} \sigma_{\mathrm{e}}{ }^{2}\right)$ em relação à avaliação que pressupõe homogeneidade de variância $\left(\mathrm{R}=\mathrm{I} \sigma_{\mathrm{e}}{ }^{2}\right)$. A característica estudada foi ganho de peso pós-desmame corrigido para 345 dias, sendo esta simulada com variância fenotípica de $300 \mathrm{~kg}^{2}$ e herdabilidade igual a 0,4 . A estrutura de um conjunto real de dados foi utilizada para fornecer os GC e os pais referentes às observações de cada animal. Cinco níveis de heterogeneidade de variância residual foram considerados de forma que os componentes de variância fossem, na média, iguais aos da situação de homogeneidade de variância. Na medida em que níveis mais acentuados de heterogeneidade de variância residual foram considerados, os animais foram selecionados dos GC com maior variabilidade, especialmente com pressão de seleção intensa. Em relação à consistência de predição, os produtos e as vacas tiveram seus valores genéticos preditos mais afetados pela heterogeneidade de variância residual do que os touros. O fator de ponderação utilizado reduziu, mas não eliminou o efeito da heterogeneidade de variância. As avaliações genéticas ponderadas apresentaram resultados iguais ou superiores àqueles obtidos pelas avaliações que assumiram homogeneidade de variância. Mesmo quando não necessário, o uso de avaliações ponderadas produziu resultados não inferiores às avaliações que assumiram homogeneidade de variância.

Palavras-chave: acurácia, consistência, estimação ponderada, simulação

\section{Effects of Heterogeneity of Residual Variance among Contemporary Groups on Genetic Evaluation of Beef Cattle}

\begin{abstract}
The objective of this study was to investigate, via simulated data, the effect of heterogeneity of residual variance among contemporary groups $(C G)$ on genetic evaluation of beef cattle, and to compare a weighted genetic evaluation procedure $\left(R \neq I \sigma_{\mathrm{e}}{ }^{2}\right)$ with one that assumes homogeneity of variances $\left(R=I \sigma_{e}{ }^{2}\right)$. Different data sets of postweaning weight gain, adjusted to 345 days, were simulated with and without heterogeneity of residual variance, using a phenotypic variance of $300 \mathrm{~kg}^{2}$ and a true heritability of 0.4 . A real data set was used to provide the CG and parents related to each animal observation. Results showed that, when high levels of heterogeneity of residual variance were considered, animals were selected from CG with higher variability, especially with intense selection. With respect to prediction consistency, non parent animals and cows had their predicted breeding values more affected by heterogeneity of residual variance than sires. The weighed factor used reduced, but did not eliminate, the effect of heterogeneity of residual variance. The results of weighted genetic evaluations were similar or superior to those from evaluations that assumed homogeneity of variances. Even when the variances were homogeneous, the weighed genetic evaluations yielded results that were not inferior than those from the usual evaluations, which assumed homogeneity of variances.
\end{abstract}

Key Words: accuracy, consistency, simulation, weighed estimate

\section{Introdução}

Na avaliação genética de bovinos, feita por meio de metodologias que geram preditores com propriedades conhecidas por BLUP (melhor preditor linear não viesado) pressupõe-se, usualmente, homogeneidade de variâncias entre os níveis dos efeitos fixos do modelo estatístico proposto. Entretanto, inúmeros trabalhos (Boldman \& Freeman, 1990; Dong \& Mao, 1990; Reverter et al., 1997; Torres, 1998) relatam a existência de heterogeneidade de variâncias genética e residual entre rebanhos, grupos de contemporâneos ou outras classificações dos efeitos ambientais.

\footnotetext{
${ }^{1}$ Parte da Dissertação de Mestrado do primeiro autor (Curso de pós-graduação em Zootecnia, área de concentração em Genética e Melhoramento Animal, Faculdade de Ciências Agrárias e Veterinárias, UNESP/Câmpus de Jaboticabal), financiada pela CAPES.

${ }^{2}$ Estudante de doutorado em Zootecnia - DZ-FCAV-UNESP-Jaboticabal, SP. Bolsista FAPESP. E.mail: rcar@fcav.unesp.br

${ }^{3}$ GenSys Consultores Associados S/C Ltda.; Lagoa da Serra Ltda. E.mail: fries@fcav.unesp.br

4 University of Guelph, Guelph, ON, Canada. E-mail: schenkel@uoguelph.ca

${ }^{5}$ Profa. Assistente Dra. DZ-FCAV-UNESP-Jaboticabal, SP. Bolsista CNPq.

E.mail: Igalb@fcav.unesp.br
} 
Quando ignorada, a heterogeneidade de variâncias genética e/ou residual pode ocasionar a redução da precisão dos valores genéticos preditos e, conseqüentemente, a redução da resposta à seleção (Garrick \& Van Vleck, 1987). De acordo com Hill (1984) e Vinson (1987), o problema causado pela heterogeneidade de variância pode ser mais grave quando ocorre apenas heterogeneidade de variância residual. Neste caso, métodos ponderados de avaliação genética seriam recomendados (Henderson, 1984).

Garrick \& Van Vleck (1987), com base em estudos de simulação de dados de produção de leite, encontraram redução de até 3,3\% no ganho genético quando a heterogeneidade de variância residual foi ignorada na avaliação genética dos animais.

Tendo em vista que a heterogeneidade de variância residual pode resultar em classificação menos precisa dos animais, prejudicando a escolha de futuros reprodutores e, conseqüentemente, o progresso genético e a rentabilidade dos rebanhos em questão, o objetivo deste trabalho foi investigar, por meio de dados simulados, o efeito da heterogeneidade de variância residual entre grupos de contemporâneos (GC) sobre as avaliações genéticas de bovinos de corte e comparar o uso de uma avaliação genética ponderada em relação à avaliação que assume homogeneidade de variância.

\section{Material e Métodos}

\section{Simulação dos dados}

Um conjunto de dados de pesos ao desmame e ao sobreano de animais da raça Nelore, fornecido pela Agropecuária Jacarezinho Ltda., foi utilizado para fornecer a estrutura dos dados, isto é, o GC e os pais referentes a cada animal. Após edição, o arquivo ficou com 12.459 produtos, distribuídos em 279 GC, filhos de 140 touros e 8.932 vacas. Este conjunto real de dados foi utilizado procurando evitar que estruturas artificiais ou distantes da realidade fossem simuladas.

Dada a estrutura dos dados, foram simulados os valores do efeito genético aditivo (a), do efeito de GC (b) e do erro aleatório (e), para gerar os ganhos de peso pós-desmame, corrigido para 345 dias (GPD345), de acordo com o modelo:

$$
\operatorname{GPD} 345=\mathrm{a}+\mathrm{b}+\mathrm{e},
$$

Optou-se pela simulação dos dados, pois tal procedimento permite uma comparação direta entre os valores simulados e as soluções geradas pelas avaliações genéticas. Para simular a, b, e e, utilizou-se a função RANNOR do SAS (SAS, 1996), que gera conjunto de valores com distribuição normal.

Inicialmente, foram simulados os valores genéticos dos touros (at) e das vacas (av) que não tinham ascendentes conhecidos, utilizando-se média igual a zero para ambos e desvios-padrão de 13,356 e 10,954 $\mathrm{kg}$, respectivamente. Houve nova simulação de at, se o mesmo fosse menor que a média menos um desviopadrão, pois, em rebanhos comerciais ou de seleção, touros com baixo valor genético dificilmente seriam utilizados como reprodutores. Após esta restrição, esperou-se que at também apresentasse desvio-padrão de 10,954 kg (Bulmer, 1971).

Dado at e av , a foi obtido por a $=((a t+a v) / 2)+$ sm, sendo sm o efeito da segregação Mendeliana dos genes, que foi simulado seguindo uma distribuição normal com média zero e desvio-padrão de $7,746 \mathrm{~kg}$. O coeficiente de endogamia não foi considerado na simulação da sm, pois apenas 11 dos 21.097 animais eram endogâmicos. Os desvios-padrão de at, av e sm foram determinados de forma que a variância genética aditiva $\left(\sigma_{\mathrm{a}}^{2}\right)$ fosse igual a $120 \mathrm{~kg}^{2}$.

Para simulação de $b$, utilizou-se média igual a 172,5 kg e desvio-padrão de 100 kg, com simulação de um novo valor se b fosse menor do que $69 \mathrm{~kg}$ ou maior do que $276 \mathrm{~kg}$ (valores estes, próximos aos da distribuição dos dados reais).

Os erros aleatórios (e) foram simulados em situações de homogeneidade e de cinco níveis de heterogeneidade de variância residual entre os GC. Em todos os casos, a média utilizada para simulação do e foi igual a zero e os desvios-padrão utilizados foram determinados de forma que as variâncias residuais médias fossem iguais a $180 \mathrm{~kg}^{2}$, resultando em uma variância fenotípica de $300 \mathrm{~kg}^{2} \mathrm{e}$, conseqüentemente, uma herdabilidade de 0,40 . Os desvios-padrão utilizados para as situações de homogeneidade $\left(\mathrm{sd}_{0}\right)$ e dos cinco níveis de heterogeneidade $\left(\operatorname{sd}_{1} \operatorname{a~sd}_{5}\right)$ de variância são ilustrados na Figura 1 e descritos a seguir:

$$
\begin{aligned}
\mathrm{sd}_{0}= & (13,416) ; \\
\mathrm{sd}_{1}= & \left(8,23957614+0,03430481 \mathrm{~b}-0,00002489 \mathrm{~b}^{2}\right) ; \\
\mathrm{sd}_{2}= & \left(0,14125552+0,09805013 \mathrm{~b}-0,00012228 \mathrm{~b}^{2}\right) ; \\
\mathrm{sd}_{3}= & \left(19,41456968-0,01367923 \mathrm{~b}-0,00012228 \mathrm{~b}^{2}\right) ; \\
\mathrm{sd}_{4}= & (17,889), \text { se }(\mathrm{b}<150 \mathrm{~kg}) \mathrm{ou}=(29,08537030 \\
& -0,09070492 \mathrm{~b}), \text { se }(150 \mathrm{~kg} \leq \mathrm{b} \leq 200 \mathrm{~kg}) \\
& \text { ou }=(6,325), \text { se }(\mathrm{b}>200 \mathrm{~kg}) ; \\
\mathrm{sd}_{5}= & \left(-2,01204693+0,85917426 \mathrm{~b}-0,00608837 \mathrm{~b}^{2}\right), \\
& \text { se }(\mathrm{b}<120 \mathrm{~kg}) \text { ou }=(22,88614517- \\
& \left.0,12316287 \mathrm{~b}+0,00022883 \mathrm{~b}^{2}\right), \operatorname{se}(\mathrm{b} \geq 120 \mathrm{~kg}),
\end{aligned}
$$

em que: b é o efeito de GC.

\section{R. Bras. Zootec., v.31, n.4, p.1680-1688, 2002}




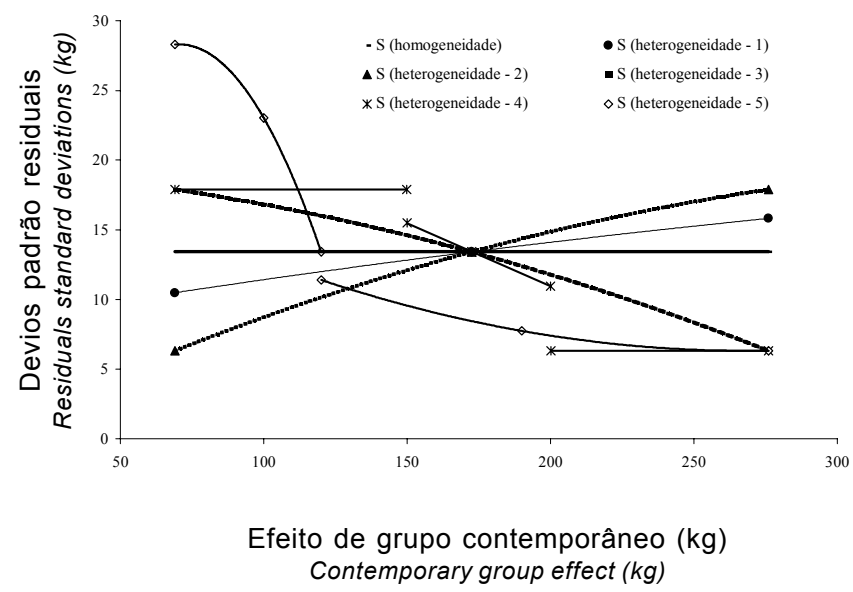

Figura 1 - Desvios padrão (S), de acordo com o efeito de GC, utilizados para simular os erros aleatórios da situação de homogeneidade de variância e dos cinco diferentes níveis de heterogeneidade de variância residual.

Figure 1 - Standard deviations (S), according to CG effect, used to simulate the random errors in the situation of homogeneity of variances and five different levels of heterogeneity of residual variance.

Os níveis 1 e 2 foram caracterizados por maiores variâncias residuais nos GC com maiores efeitos, ocorrendo o inverso nos níveis 3,4 e 5 .

Os efeitos ambientais e genéticos foram simulados de forma a serem não correlacionados, a fim de testar exclusivamente o efeito de heterogeneidade de variância.

Após o processo de simulação, ainda foram feitas restrições finais sobre GPD345, de modo que tal característica variasse dentro do intervalo de 34,5 e $310,5 \mathrm{~kg}$.

Seguindo todas as condições mencionadas anteriormente, foram simulados 100 conjuntos de dados (repetições) para cada uma das situações (com e sem heterogeneidade de variância), procurando, com isso, maior segurança nas inferências. Os valores dos efeitos de GC foram simulados apenas uma vez e depois mantidos nas demais repetições (caracterizando a condição de efeito fixo).

\section{Avaliações genéticas}

Cada conjunto de dados foi submetido à estimação dos efeitos de GC e predição dos valores genéticos, seguindo a metodologia de modelos mistos com o emprego do modelo de touro e vaca, conforme descrito em Schaeffer (1993), pressupondo homogeneidade de variâncias, ou seja, considerando inicialmente a matriz de (co)variância residual (R) igual ao produto de uma matriz identidade (I) pela variância residual $\left(\sigma_{\mathrm{e}}^{2}\right)$, isto é, $\mathrm{R}=\mathrm{I} \sigma_{\mathrm{e}}^{2}$.

Posteriormente, os conjuntos de dados foram submetidos à avaliação genética ponderada, considerando a matriz $\mathrm{R} \neq \mathrm{I} \sigma_{\mathrm{e}}{ }^{2}$, por meio de um fator de ponderação (RPOND), sendo RPOND $=\sigma_{\mathrm{m}} / \sigma_{\mathrm{GC}}$, em que: $\sigma_{\mathrm{m}}=$ desvio-padrão médio dos $\mathrm{GC} ; \sigma_{\mathrm{GC}}=$ desvio-padrão do GC específico (porém, se $\sigma_{\mathrm{GC}}<\sigma_{\mathrm{m}}$, então $\sigma_{\mathrm{GC}}=\sigma_{\mathrm{m}}$ ). Buscou-se através do RPOND dar menor importância às observações pertencentes a GC com maiores $\sigma_{\mathrm{e}}^{2}$, procurando, ao mesmo tempo, não supervalorizar observações de GC com $\sigma_{\mathrm{e}}{ }^{2} \mathrm{ex}$ tremamente baixas. $\mathrm{O}$ fator de ponderação utilizado (RPOND) é uma simplificação do fator de ponderação robusto (ROB) descrito por Schenkel \& Brito (1994).

Para ambos os modelos, as soluções para os animais sem progênie foram obtidas por retro-substituição. As variâncias consideradas nas avaliações genéticas foram iguais às utilizadas no processo de simulação.

\section{Critérios para comparação dos resultados}

Para as estimativas dos contrastes de GC e para os valores genéticos preditos dos produtos, touros e vacas, foram calculadas as correlações médias de Pearson, das 100 repetições, entre tais valores e os valores simulados, para cada situação, como uma medida da acurácia de predição ou estimação.

Objetivando uma outra forma de comparação, foram obtidas as médias dos desvios absolutos médios (DAM) dos valores preditos em relação aos valores simulados. DAM é uma medida da consistência do preditor, isto é, da proximidade do valor predito em relação ao parâmetro verdadeiro, dado o tamanho da amostra. As DAM foram definidas como: DAM $=\frac{1}{q} \sum_{i=1}^{q}$ $\operatorname{abs}\left(\hat{\mathrm{a}}_{\mathrm{i}}-\mathrm{a}_{\mathrm{i}}\right)$, onde: $\mathrm{q}$ é o número de produtos, touros ou vacas, e abs refere-se ao valor absoluto da diferença entre o valor genético aditivo predito $\left(\hat{\mathrm{a}}_{\mathrm{i}}\right)$ e o valor genético aditivo verdadeiro $\left(a_{i}\right)$.

As DAM também foram obtidas por classe de efeito de GC, assim como as porcentagens médias dos produtos selecionados sob diferentes pressões de seleção. Foram definidas três classes de efeito de GC (b): (1) $\mathrm{b}<150 \mathrm{~kg}$ (4.584 animais distribuídos em 108 GC); (2) $150 \mathrm{~kg} \leq \mathrm{b} \leq 200 \mathrm{~kg}$ (3.162 animais distribuídos em $69 \mathrm{GC})$; e (3) b > $200 \mathrm{~kg}$ (4.713 animais distribuídos em 102 GC).

Finalmente, foram obtidas as DAM e as médias dos valores genéticos verdadeiros dos touros $10 \%$ superiores e das vacas e produtos $10 \%$ e $1 \%$ superiores.

\section{R. Bras. Zootec., v.31, n.4, p.1680-1688, 2002}




\section{Resultados e Discussão}

As correlações amostrais médias, das 100 repetições, entre os valores verdadeiros e os valores estimados ou preditos (acurácias médias) são apresentadas na Tabela 1. As correlações entre os contrastes estimados e os efeitos simulados de GC foram altas $(0,998)$ e iguais nas diferentes situações simuladas. Pode-se perceber que para os valores genéticos preditos, praticamente não houve redução das correlações em nenhum dos níveis de heterogeneidade de variância residual em comparação com a situação de homogeneidade de variância, isto é, a heterogeneidade não afetou a acurácia de estimação e de predição.

As correlações obtidas através da avaliação genética ponderada foram sempre iguais ou superiores àquelas obtidas quando se ignorou a heterogeneidade de variância. Os maiores aumentos observados ao utilizar o fator de ponderação ocorreram no nível $5 \mathrm{de}$ heterogeneidade de variância, sobretudo para as vacas. Neste caso, o baixo número médio de progênies por vaca $(1,39)$ pode estar associado ao resultado obtido.
Na Tabela 2, são fornecidas as médias dos desvios absolutos médios (DAM) entre os valores genéticos verdadeiros e preditos. Menores DAM indicam maior consistência de predição. Para os produtos e vacas as DAM foram maiores, o que era esperado uma vez que os produtos têm dados em apenas um GC e as vacas apresentam, em comparação com os touros, menor quantidade de dados referentes às suas progênies. Com base nos resultados da Tabela 2, assim como nos da Tabela 1, praticamente não foi possível observar o efeito da heterogeneidade de variância residual entre GC.

Tendo em vista os resultados anteriores, procurou-se avaliar o efeito da heterogeneidade de variância residual sobre as DAM, por classe de efeito de GC (Tabela 3). É possível verificar que o efeito da heterogeneidade de variância residual foi distinto nas diferentes classes. Em todos os níveis de heterogeneidade de variância, os valores genéticos preditos dos produtos foram mais próximos aos valores verdadeiros (menores DAM) para os animais com dados nos GC com menores variâncias residuais. O

Tabela 1 - Correlações médias de Pearson, das 100 repetições, entre os valores genéticos verdadeiros e preditos de touros, vacas e produtos e entre os contrastes estimados e os efeitos simulados de GC, nas situações de homogeneidade e de cinco níveis de heterogeneidade de variância residual

Table 1 - Averages of Pearson correlation coefficient, from100 replicates, among true and predicted genetic values of sires, dams and non parent animals and among estimated and simulated contrasts between CG effects, in situations of homogeneity and five different levels of heterogeneity of residual variance

\begin{tabular}{|c|c|c|c|c|c|c|}
\hline $\begin{array}{l}\text { Categoria } \\
\text { Category }\end{array}$ & & & & $\begin{array}{l}\text { Situação } \\
\text { Situation }\end{array}$ & & \\
\hline $\begin{array}{l}\text { Avaliação Genética }{ }^{1} \\
\text { Genetic evaluation }^{1}\end{array}$ & $\begin{array}{l}\text { Homog. } \\
\text { Homogeneity }\end{array}$ & $\begin{array}{l}\text { Heterog. } \\
\text { (nível 1) } \\
\text { Heterogeneity } \\
\text { (level 1) }\end{array}$ & $\begin{array}{l}\text { Heterog. } \\
\text { (nível 2) } \\
\text { Heterogeneity } \\
\text { (level 2) }\end{array}$ & $\begin{array}{l}\text { Heterog. } \\
\text { (nível 3) } \\
\text { Heterogeneity } \\
\text { (level 3) }\end{array}$ & $\begin{array}{l}\text { Heterog. } \\
\text { (nível 4) } \\
\text { Heterogeneity } \\
\text { (level 4) }\end{array}$ & $\begin{array}{l}\text { Heterog. } \\
\text { (nível 5) } \\
\text { Heterogeneity } \\
\text { (level 5) }\end{array}$ \\
\hline Touro & & & & & & \\
\hline Sire & 0,870 & 0,869 & 0,867 & 0,873 & 0,874 & 0,880 \\
\hline $\begin{array}{l}2 \\
\text { Vaca } \\
\text { Dam }\end{array}$ & 0,870 & 0,869 & 0,869 & 0,876 & 0,879 & 0,893 \\
\hline $\begin{array}{l}1 \\
2\end{array}$ & $\begin{array}{l}0,372 \\
0,372\end{array}$ & $\begin{array}{l}0,371 \\
0,372\end{array}$ & $\begin{array}{l}0,370 \\
0,375\end{array}$ & $\begin{array}{l}0,376 \\
0,381\end{array}$ & $\begin{array}{l}0,376 \\
0,390\end{array}$ & $\begin{array}{l}0,382 \\
0,423\end{array}$ \\
\hline $\begin{array}{l}\text { Produto } \\
\text { Non parent animal }\end{array}$ & & & & & & \\
\hline $\begin{array}{l}1 \\
2 \\
\text { GC }\end{array}$ & $\begin{array}{l}0,701 \\
0,701\end{array}$ & $\begin{array}{l}0,700 \\
0,700\end{array}$ & $\begin{array}{l}0,699 \\
0,701\end{array}$ & $\begin{array}{l}0,703 \\
0,705\end{array}$ & $\begin{array}{l}0,703 \\
0,708\end{array}$ & $\begin{array}{l}0,709 \\
0,722\end{array}$ \\
\hline$C G$ & & & & & & \\
\hline 1 & 0,998 & 0,998 & 0,998 & 0,998 & 0,998 & 0,998 \\
\hline 2 & 0,998 & 0,998 & 0,998 & 0,998 & 0,998 & 0,998 \\
\hline
\end{tabular}

1 Avaliação genética 1 : considerando a matriz $\mathrm{R}=1 \sigma_{\mathrm{e}}^{2}$.

Avaliação genética 2: utilizando o fator de ponderação RPOND.

1 Genetic evaluation 1: considering $R=/ \sigma_{e}{ }^{2}$.

Genetic evaluation 2: using the RPOND weighted factor.

R. Bras. Zootec., v.31, n.4, p.1680-1688, 2002 
Tabela 2 - Médias, das 100 repetições, dos desvios absolutos médios (DAM ${ }^{1}$ ) entre os valores genéticos verdadeiros e preditos de touros, vacas e produtos, nas situações de homogeneidade e de cinco níveis de heterogeneidade de variância residual

Table 2 - Averages, from 100 replicates, of the mean absolute deviations $\left(M A D^{1}\right)$ among true and predicted genetic values of sires, dams and non parent animals, in situations of homogeneity and five different levels of heterogeneity of residual variance

\begin{tabular}{|c|c|c|c|c|c|c|}
\hline $\begin{array}{l}\text { Categoria } \\
\text { Category }\end{array}$ & & & & $\begin{array}{l}\text { Situação } \\
\text { Situation }\end{array}$ & & \\
\hline $\begin{array}{l}\text { Avaliação Genética }{ }^{1} \\
\text { Genetic evaluation }^{1}\end{array}$ & $\begin{array}{l}\text { Homog. } \\
\text { Homogeneity }\end{array}$ & $\begin{array}{c}\text { Heterog. } \\
\text { (nível 1) } \\
\text { Heterogeneity } \\
\text { (level 1) }\end{array}$ & $\begin{array}{l}\text { Heterog. } \\
\text { (nível 2) } \\
\text { Heterogeneity } \\
\text { (level 2) }\end{array}$ & $\begin{array}{c}\text { Heterog. } \\
\text { (nível 3) } \\
\text { Heterogeneity } \\
\text { (level 3) }\end{array}$ & $\begin{array}{c}\text { Heterog. } \\
\text { (nível 4) } \\
\text { Heterogeneity } \\
\text { (level 4) }\end{array}$ & $\begin{array}{c}\text { Heterog. } \\
\text { (nível 5) } \\
\text { Heterogeneity } \\
\text { (level 5) }\end{array}$ \\
\hline $\begin{array}{l}\text { Touro } \\
\text { Sire }\end{array}$ & & & & & & \\
\hline $\begin{array}{l}1 \\
2 \\
\text { Vaca }\end{array}$ & $\begin{array}{l}5,16 \\
5,16\end{array}$ & $\begin{array}{l}5,17 \\
5,16\end{array}$ & $\begin{array}{l}5,19 \\
5,16\end{array}$ & $\begin{array}{l}5,12 \\
5,09\end{array}$ & $\begin{array}{l}5,11 \\
5,04\end{array}$ & $\begin{array}{l}5,03 \\
4,87\end{array}$ \\
\hline $\begin{array}{l}\text { Dam } \\
1 \\
2\end{array}$ & $\begin{array}{l}8,12 \\
8,12\end{array}$ & $\begin{array}{l}8,12 \\
8,12\end{array}$ & $\begin{array}{l}8,12 \\
8,11\end{array}$ & $\begin{array}{l}8,10 \\
8,09\end{array}$ & $\begin{array}{l}8,10 \\
8,05\end{array}$ & $\begin{array}{l}8,07 \\
7,96\end{array}$ \\
\hline $\begin{array}{l}\text { Produto } \\
\text { Non parent animal }\end{array}$ & & & & & & \\
\hline $\begin{array}{l}1 \\
2 \\
\end{array}$ & $\begin{array}{l}6,42 \\
6,42\end{array}$ & $\begin{array}{l}6,42 \\
6,42 \\
\end{array}$ & $\begin{array}{l}6,42 \\
6,40\end{array}$ & $\begin{array}{l}6,38 \\
6,37 \\
\end{array}$ & $\begin{array}{l}6,36 \\
6,32 \\
\end{array}$ & $\begin{array}{l}6,27 \\
6,18\end{array}$ \\
\hline
\end{tabular}

1 Valores em kg.

2 Avaliação genética 1: considerando a matriz $R=l \sigma_{e}{ }^{2}$.

Avaliação genética 2: utilizando o fator de ponderação RPOND.

1 Values in $\mathrm{kg}$.

${ }^{2}$ Genetic evaluation 1: considering $R=1 \sigma_{e}^{2}$.

Genetic evaluation 2: using the RPOND weighted factor.

oposto foi verdadeiro para os GC com as maiores variâncias residuais, os quais apresentarammaiores DAM.

Cabe salientar que, no processo de simulação, os níveis 1 e 2 de heterogeneidade de variância foram caracterizados por maiores variâncias residuais nos GC com maiores efeitos. Já, nos níveis 3, 4 e 5 as variâncias residuais são maiores para os $\mathrm{GC}$ com menores efeitos sobre o GPD345, conforme ilustrado na Figura 1.

No geral, os níveis 2 e 3 apresentaram resultados semelhantes (porém opostos em relação aos efeitos de GC) evidenciando que o sentido da associação entre a heterogeneidade e os efeitos de GC, praticamente, não alterou o efeito da heterogeneidade de variância residual.

De acordo com a Tabela 3 , o efeito da heterogeneidade de variância residual nas avaliações (efeito favorável nas classes de baixa variância residual e desfavorável nas classes de alta variância residual) não foi notado quando comparações totais dos dados foram realizadas, devido à uma "compensação" entre as DAM dos GC com menores e maiores variâncias residuais.
Os níveis de heterogeneidade de variância mais acentuados apresentaram maiores amplitudes das DAM, entre as classes de baixo e alto efeito de GC, sendo que para a situação de homogeneidade de variância praticamente não existiram diferenças entre as DAM. A utilização da avaliação genética ponderada fez com que resultados iguais ou melhores fossem obtidos, em relação à avaliação que ignorou a heterogeneidade de variância residual (Tabela 3 ).

Procurando ainda avaliar o efeito da heterogeneidade de variância residual sobre a consistência de predição dos valores genéticos, utilizando a DAM como critério de avaliação, foram investigados os resultados referentes aos touros, vacas e produtos sob diferentes pressões de seleção (Tabela 4).

Para touros, foi utilizada apenas pressão de seleção de $10 \%$, devido à quantidade de animais nesta categoria. Os níveis 4 e 5 de heterogeneidade de variância resultaram em pequenos aumentos das DAM dos touros. As predições dos valores genéticos das vacas e produtos foram mais afetadas pela heterogeneidade de variância que os valores dos touros. Nos níveis 2 a 5, com pressão de seleção de 
Tabela 3 - Médias, das 100 repetições, dos desvios absolutos médios (DAM ${ }^{1}$ ) entre os valores genéticos verdadeiros e preditos dos produtos, por classe de efeito de GC (b), nas situações de homogeneidade e de cinco níveis de heterogeneidade de variância residual

Table 3 - Averages, from 100 replicates, of the mean absolute deviations (MAD ${ }^{1}$ ) among true and predicted genetic values of non parent animals, per CG class effect (b), in situations of homogeneity and five different levels of heterogeneity of residual variance

\begin{tabular}{|c|c|c|c|c|c|c|}
\hline \multirow[b]{2}{*}{$\begin{array}{l}\text { Avaliação genética }{ }^{1} \\
\text { Genetic evaluation }^{1} \\
\quad \text { (Classe) } \\
\quad \text { (Class) }\end{array}$} & \multirow[b]{2}{*}{$\begin{array}{l}\text { Homog. } \\
\text { Homogeneity }\end{array}$} & \multicolumn{5}{|c|}{$\begin{array}{l}\text { Situação } \\
\text { Situation }\end{array}$} \\
\hline & & $\begin{array}{l}\text { Heterog. } \\
\text { (nível 1) } \\
\text { Heterogeneity } \\
\text { (level 1) }\end{array}$ & $\begin{array}{l}\text { Heterog. } \\
\text { (nível 2) } \\
\text { Heterogeneity } \\
\text { (level 2) }\end{array}$ & $\begin{array}{l}\text { Heterog. } \\
\text { (nível 3) } \\
\text { Heterogeneity } \\
\text { (level 3) }\end{array}$ & $\begin{array}{l}\text { Heterog. } \\
\text { (nível 4) } \\
\text { Heterogeneity } \\
\text { (level 4) }\end{array}$ & $\begin{array}{l}\text { Heterog. } \\
\text { (nível 5) } \\
\text { Heterogeneity } \\
\text { (level 5) }\end{array}$ \\
\hline \multicolumn{7}{|l|}{$\mathrm{R}=\mathrm{I} \sigma_{\mathrm{e}}^{2}$} \\
\hline$(b<150 \mathrm{~kg})$ & 6,41 & 6,18 & 5,92 & 6,85 & 7,12 & 7,25 \\
\hline$(150 \leq b \leq 200 \mathrm{~kg})$ & 6,43 & 6,45 & 6,46 & 6,39 & 6,38 & 5,79 \\
\hline$(b>200 \mathrm{~kg})$ & 6,43 & 6,65 & 6,88 & 5,92 & 5,61 & 5,65 \\
\hline Total & 6,42 & 6,42 & 6,42 & 6,38 & 6,36 & 6,27 \\
\hline \multicolumn{7}{|l|}{ RPOND } \\
\hline$(b<150 \mathrm{~kg})$ & 6,41 & 6,18 & 5,90 & 6,82 & 7,07 & 7,08 \\
\hline$(150 \leq b \leq 200 \mathrm{~kg})$ & 6,43 & 6,44 & 6,46 & 6,39 & 6,37 & 5,75 \\
\hline$(b>200 \mathrm{~kg})$ & 6,43 & 6,64 & 6,85 & 5,91 & 5,57 & 5,59 \\
\hline Total & 6,42 & 6,42 & 6,40 & 6,37 & 6,32 & 6,18 \\
\hline Total & & & & & & \\
\hline
\end{tabular}

${ }^{1}$ Valores em kg.

${ }^{2}$ Avaliação genética considerando a matriz $\mathrm{R}=1 \sigma_{\mathrm{e}}{ }^{2}$ ou utilizando o fator de ponderação $R P O N D$.

1 Values in $\mathrm{kg}$.

2 Genetic evaluation considering $R=l \sigma_{e}{ }^{2}$ or using the RPOND weighted factor.

$10 \%$, houve aumentos significativos das DAM destas categorias, indicando perdas na consistência das predições. $\mathrm{O}$ aumento da pressão de seleção resultou em maiores efeitos da heterogeneidade de variância residual nos níveis 4 e 5 , para as vacas, e em todos os níveis para os produtos. No nível mais elevado de heterogeneidade de variância (5) e com alta pressão de seleção, o uso do RPOND reduziu substancialmente a DAM das vacas e produtos (Tabela 4).

Estes resultados evidenciam que, a partir de determinada pressão de seleção, a "compensação" das DAM obtidas das classes de diferentes variâncias, citada anteriormente (Tabela 3), não ocorre de forma efetiva como quando comparações totais dos dados são realizadas.

O efeito de maior seleção de indivíduos dos grupos mais variáveis pode ser observado na Tabela 5. No nível 2 de heterogeneidade de variância, por exemplo, houve aumento de $15,7 \%$ e de $41,8 \%$ na proporção de produtos selecionados (com pressões de seleção de $10 \%$ e $1 \%$, respectivamente) da classe de maior efeito de GC, em comparação com a mesma classe na situação de homogeneidade de variância.

Assim como demonstrado em Hill (1984) e Vinson (1987), quanto maior a pressão de seleção (menos indivíduos selecionados), maiores foram as propor- ções de indivíduos selecionados das classes mais variáveis.

De acordo com os resultados encontrados por Meuwissen \& Van Der Werf (1993), à medida que níveis mais acentuados de heterogeneidade de variâncias foram simulados, a proporção de animais selecionados dos grupos mais variáveis também foi aumentada.

Segundo Hill (1984), a tendência de selecionar maior quantidade de indivíduos dos grupos mais variáveis seria um procedimento favorável se a heterogeneidade de variância fosse resultante de fatores genéticos. No entanto, como, no presente estudo, foi simulada apenas heterogeneidade de variância residual, a maior seleção de animais dos GC mais variáveis não seria desejada, pois, numa aplicação prática, animais de GC com os efeitos ambientais menos "controlados" seriam beneficiados.

Os resultados da Tabela 5 indicam que, apesar de o fator de ponderação utilizado ter se mostrado pouco eficiente, seu uso, no geral, reduziu a tendência de selecionar mais animais das classes com maior variância residual.

Finalmente, como critério de comparação dos resultados, foram obtidas as médias dos valores genéticos verdadeiros (vga) dos touros, vacas e produtos selecio- 
Tabela 4 - Médias, das 100 repetições, dos desvios absolutos médios (DAM ${ }^{1}$ ) entre os valores genéticos verdadeiros e preditos de touros, vacas e produtos selecionados com diferentes pressões de seleção ${ }^{2}$, nas situações de homogeneidade e de cinco níveis de heterogeneidade de variância residual

Table 4 - Averages, from 100 replicates, of the mean absolute deviations $\left(M A D^{1}\right)$ among true and predicted genetic values of sires, dams and non parent animals selected under different proportions saved ${ }^{2}$, in situation of homogeneity and five different levels of heterogeneity of variance

\begin{tabular}{|c|c|c|c|c|c|c|}
\hline $\begin{array}{l}\text { Categoria } \\
\text { Category }\end{array}$ & & & & $\begin{array}{l}\text { Situação } \\
\text { Situation }\end{array}$ & & \\
\hline $\begin{array}{l}\text { Avaliação genética } 1 \\
\text { Genetic evaluation }^{1}\end{array}$ & $\begin{array}{l}\text { Homog. } \\
\text { Homogeneity }\end{array}$ & $\begin{array}{l}\text { Heterog. } \\
\text { (nível 1) } \\
\text { Heterogeneity } \\
\text { (level 1) }\end{array}$ & $\begin{array}{l}\text { Heterog. } \\
\text { (nível 2) } \\
\text { Heterogeneity } \\
\text { (level 2) }\end{array}$ & $\begin{array}{l}\text { Heterog. } \\
\text { (nível 3) } \\
\text { Heterogeneity } \\
\text { (level 3) }\end{array}$ & $\begin{array}{l}\text { Heterog. } \\
\text { (nível 4) } \\
\text { Heterogeneity } \\
\text { (level 4) }\end{array}$ & $\begin{array}{l}\text { Heterog. } \\
\text { (nível 5) } \\
\text { Heterogeneity } \\
\text { (level 5) }\end{array}$ \\
\hline Touro & & & & & & \\
\hline Sire & 5,40 & 5,41 & 5,38 & 5,40 & 5,45 & 5,58 \\
\hline $\begin{array}{l}2 \\
\text { Vaca }\end{array}$ & 5,40 & 5,37 & 5,34 & 5,39 & 5,40 & 5,50 \\
\hline $\begin{array}{l}\text { Dam } \\
1 \\
2\end{array}$ & $\begin{array}{l}8,00(7,78) \\
8,00(7,76)\end{array}$ & $\begin{array}{l}8,03(7,81) \\
8,01(7,78)\end{array}$ & $\begin{array}{l}8,12(8,00) \\
8,07(7,86)\end{array}$ & $\begin{array}{l}8,10(8,08) \\
8,05(7,88)\end{array}$ & $\begin{array}{l}8,28(8,37) \\
8,17(8,04)\end{array}$ & $\begin{array}{l}8,60(9,84) \\
8,16(8,15)\end{array}$ \\
\hline $\begin{array}{l}\text { Produto } \\
\text { Non parent animal } \\
1 \\
2\end{array}$ & $\begin{array}{l}6,44(6,52) \\
6,44(6,52)\end{array}$ & $\begin{array}{l}6,48(6,62) \\
6,48(6,62)\end{array}$ & $\begin{array}{l}6,62(6,85) \\
6,60(6,85)\end{array}$ & $\begin{array}{l}6,63(6,91) \\
6,60(6,88)\end{array}$ & $\begin{array}{l}6,89(7,38) \\
6,85(7,30)\end{array}$ & $\begin{array}{l}7,45(9,67) \\
7,26(8,91)\end{array}$ \\
\hline $\begin{array}{l}1{ }^{1} \text { Valores em kg. } \\
2 \text { Valores entre ( ) são } \\
{ }^{3} \text { Avaliação genética } 1: \\
\text { Avaliação genética } 2: \\
1 \text { Values in kg. } \\
{ }^{2} \text { Values between () are re } \\
{ }^{3} \text { Genetic evaluation 1: con } \\
\text { Genetic evaluation 2: usir }\end{array}$ & $\begin{array}{l}\text { à pressão } \\
\text { ando a matriz } \\
\text { lo o fator de } \\
\text { a proportion of } \\
=I \sigma_{e}^{2} \text {. } \\
\text { OND weighted }\end{array}$ & $\begin{array}{l}\text { eleção de } 1 \% \\
l \sigma_{e}^{2} \\
\text { eração RPONL } \\
\text { f selected animal }\end{array}$ & demais, $10 \%$ & $10 \%$ & & \\
\hline
\end{tabular}

nados com diferentes pressões de seleção, procurando, com isto, avaliar se as perdas em consistência das predições (ilustradas na Tabela 4) resultariam, conseqüentemente, em redução do ganho genético. As médias dos vga dos touros (10\% superiores) foram semelhantes nas diferentes situações simuladas (Tabela 6). Em relação às médias dos vga das vacas, não foram observadas reduções no nível 1 de heterogeneidade, em ambas pressões de seleção. No geral, os demais níveis apresentaram reduções das médias dos vga, sobretudo com pressão de seleção de 1\%. As DAM encontradas nas predições dos valores genéticos dos produtos, com pressão de seleção de $10 \%$ (Tabela 4), não refletiram em menores médias dos vga (Tabela 6), para estes animais. Já, considerando pressão de seleção de $1 \%$, ocorreram reduções significativas de tais valores com a existência de heterogeneidade de variância residual (reduções de 1,0; 3,1; 1,7; 5,3 e 16,6\%, para os níveis 1 , 2, 3, 4 e 5, respectivamente).

Garrick \& Van Vleck (1987), por meio de estudo de simulação, também encontraram redução na resposta à seleção, quando a heterogeneidade de variância foi ig- norada na avaliação genética dos animais. Meuwissen \& Van Der Werf (1993), apesar de terem encontrado maior seleção de animais pertencentes a grupos mais variáveis e viés na predição dos valores genéticos de fêmeas elite, relataram uma tendência de aumento no ganho genético quando apenas heterogeneidade de variância genética foi simulada. Entretanto, os autores não simularam situações de heterogeneidade de variância apenas residual.

O uso do RPOND, nos diferentes níveis de heterogeneidade de variância residual e em todas categorias, resultou em aumento da média dos vga, ou seja, nestas condições seria esperada maior resposta à seleção, se avaliações genéticas ponderadas fossem utilizadas. A eficiência das avaliações genéticas ponderadas frente à heterogeneidade de variância residual foi mais marcante para as vacas, evidenciando a importância da estrutura dos dados e sua associação com os resultados obtidos, uma vez que, no conjunto de dados utilizado, as vacas apresentaram, em média, poucos filhos. No nível 5 de heterogeneidade, sob pressão de seleção de $1 \%$, a média dos vga das 
vacas aumentou de $8,23 \mathrm{~kg}$ para $12,16 \mathrm{~kg}$ com o uso do RPOND, evidenciando perdas significativas na resposta à seleção se a heterogeneidade de variância residual for ignorada (Tabela 6).

Novos estudos, testando e propondo outros fatores de ponderação são recomendados.

Tabela 5 - Porcentagens médias, das 100 repetições, de produtos selecionados por classe de efeito de GC (b), com pressões de seleção ${ }^{1}$ de $10 \%$ e $1 \%$, nas situações de homogeneidade e de cinco níveis de heterogeneidade de variância residual

Table 5 - Average percentage of selected animals (over 100 replicates), per CG class effect (b), considering different proportions saved ${ }^{1}$, in situation of homogeneity and five different levels of heterogeneity of residual variance

\begin{tabular}{|c|c|c|c|c|c|c|}
\hline \multirow[b]{2}{*}{$\begin{array}{l}\text { Avaliação genética }{ }^{1} \\
\text { Genetic evaluation }^{1} \\
\quad \text { (Classe) } \\
\text { (Class) }\end{array}$} & \multirow[b]{2}{*}{$\begin{array}{l}\text { Homog. } \\
\text { Homogeneity }\end{array}$} & \multicolumn{5}{|c|}{$\begin{array}{l}\text { Situação } \\
\text { Situation }\end{array}$} \\
\hline & & $\begin{array}{c}\text { Heterog. } \\
\text { (nível 1) } \\
\text { Heterogeneity } \\
\text { (level 1) }\end{array}$ & $\begin{array}{l}\text { Heterog. } \\
\text { (nível 2) } \\
\text { Heterogeneity } \\
\text { (level 2) } \\
\end{array}$ & $\begin{array}{c}\text { Heterog. } \\
\text { (nível 3) } \\
\text { Heterogeneity } \\
\text { (level 3) } \\
\end{array}$ & $\begin{array}{c}\text { Heterog. } \\
\text { (nível 4) } \\
\text { Heterogeneity } \\
\text { (level 4) }\end{array}$ & $\begin{array}{c}\text { Heterog. } \\
\text { (nível 5) } \\
\text { Heterogeneity } \\
\text { (level 5) }\end{array}$ \\
\hline \multicolumn{7}{|l|}{$\mathrm{R}=\mathrm{I} \sigma_{e}^{2}$} \\
\hline$(b<150 \mathrm{~kg})$ & $36,9(36,7)$ & $34,0(29,0)$ & $30,6(21,1)$ & $43,0(53,1)$ & $46,8(63,8)$ & $49,3(74,6)$ \\
\hline$(150 \leq b \leq 200 \mathrm{~kg})$ & $25,0(24,1)$ & $25,0(23,6)$ & $25,3(23,3)$ & $25,0(23,0)$ & $25,2(21,9)$ & $20,8(11,2)$ \\
\hline$(b>200 \mathrm{~kg})$ & $38,1(39,2)$ & $41,0(47,4)$ & $44,1(55,6)$ & $32,0(23,9)$ & $28,0(14,3)$ & $29,9(14,2)$ \\
\hline RPOND & & & & & & \\
\hline$(b<150 \mathrm{~kg})$ & $36,9(36,9)$ & $34,3(29,6)$ & $31,3(22,4)$ & $42,3(51,3)$ & $45,5(60,8)$ & $47,5(69,3)$ \\
\hline$(150 \leq b \leq 200 \mathrm{~kg})$ & $25,0(23,4)$ & $25,1(23,7)$ & $25,4(24,2)$ & $25,2(23,7)$ & $25,6(23,2)$ & $21,5(13,8)$ \\
\hline$(b>200 \mathrm{~kg})$ & $38,1(39,7)$ & $40,6(46,7)$ & $43,3(53,4)$ & $32,5(25,0)$ & $28,9(16,0)$ & $31,0(16,9)$ \\
\hline
\end{tabular}

1 Valores entre ( ) são referentes à pressão de seleção de $1 \%$ e, os demais, $10 \%$.

1 Values between () are related to a proportion of $1 \%$ of selected animals, and the other values, $10 \%$.

Tabela 6 - Médias ${ }^{1}$, das 100 repetições, dos valores genéticos verdadeiros dos touros, vacas e produtos selecionados com diferentes pressões de seleção ${ }^{2}$, nas situações de homogeneidade e de cinco níveis de heterogeneidade de variância residual

Table 6 - Averages ${ }^{1}$, from 100 replicates, of the true genetic values of sires, dams and non parent animals selected under different proportions saved 2 in situations of homogeneity and five different levels of heterogeneity of residual variance

\begin{tabular}{|c|c|c|c|c|c|c|}
\hline \multirow{2}{*}{$\begin{array}{l}\text { Categoria } \\
\text { Category } \\
\text { Avaliação genética } 1^{1} \\
\text { Genetic evaluation }{ }^{1}\end{array}$} & \multirow[b]{2}{*}{$\begin{array}{l}\text { Homog. } \\
\text { Homogeneity }\end{array}$} & \multicolumn{5}{|c|}{$\begin{array}{l}\text { Situação } \\
\text { Situation }\end{array}$} \\
\hline & & $\begin{array}{l}\text { Heterog. } \\
\text { (nível 1) } \\
\text { Heterogeneity } \\
\text { (level 1) }\end{array}$ & $\begin{array}{l}\text { Heterog. } \\
\text { (nível 2) } \\
\text { Heterogeneity } \\
\text { (level 2) }\end{array}$ & $\begin{array}{c}\text { Heterog. } \\
\text { (nível 3) } \\
\text { Heterogeneity } \\
\text { (level 3) }\end{array}$ & $\begin{array}{l}\text { Heterog. } \\
\text { (nível 4) } \\
\text { Heterogeneity } \\
\text { (level 4) }\end{array}$ & $\begin{array}{l}\text { Heterog. } \\
\text { (nível 5) } \\
\text { Heterogeneity } \\
\text { (level 5) }\end{array}$ \\
\hline \multicolumn{7}{|l|}{ Touro } \\
\hline \multicolumn{7}{|l|}{ Sire } \\
\hline 1 & 22,46 & 22,39 & 22,36 & 22,47 & 22,43 & 22,48 \\
\hline 2 & 22,46 & 22,42 & 22,41 & 22,50 & 22,52 & 22,79 \\
\hline \multirow{2}{*}{\multicolumn{7}{|c|}{$\begin{array}{l}\text { Vaca } \\
\text { Dam }\end{array}$}} \\
\hline & & & & & & \\
\hline 1 & $7,32(11,62)$ & $7,30(11,62)$ & $7,24(11,59)$ & $7,25(11,27)$ & $7,03(10,63)$ & $7,12(8,23)$ \\
\hline 2 & $7,32(11,63)$ & $7,30(11,66)$ & $7,34(11,96)$ & $7,42(11,84)$ & $7,41(11,46)$ & $8,06(12,16)$ \\
\hline \multicolumn{7}{|l|}{ Produto } \\
\hline $\begin{array}{l}\text { Non parent animal } \\
1\end{array}$ & $15,66(23,07)$ & $15,64(22,83)$ & $15,59(22,36)$ & $15,66(22,68)$ & $15,58(21,84)$ & $15,54(19,25)$ \\
\hline 2 & $15,67(23,13)$ & $15,65(22,92)$ & $15,65(22,53)$ & $15,71(22,75)$ & $15,67(22,13)$ & $15,82(20,47)$ \\
\hline
\end{tabular}

${ }^{1}$ Valores em kg (Values in $\mathrm{kg}$ ).

2 Valores entre ( ) são referentes à pressão de selecão de $1 \%$ e, as demais, $10 \%$.

${ }^{3}$ Avaliação Genética 1: considerando a matriz $R=\mid \sigma_{e}{ }^{2}$.

Avaliação Genética 2: utilizando o fator de ponderação RPOND.

${ }^{2}$ Values between ( ) are related to a proportion of $1 \%$ of selected animals, and the other values, $10 \%$

${ }^{3}$ Genetic evaluation 1: considering $R=l \sigma_{e}^{2}$.

Genetic evaluation 2: using the RPOND weighted factor.

R. Bras. Zootec., v.31, n.4, p.1680-1688, 2002 


\section{Conclusões}

Os efeitos da heterogeneidade de variância residual sobre as avaliações genéticas estão associados à pressão de seleção e aos níveis de heterogeneidade. Se correções não forem feitas, rebanhos que praticam seleção intensa e que apresentam níveis acentuados de heterogeneidade de variância residual podem ter seus animais classificados de maneira incorreta e, conseqüentemente, menor resposta à seleção.

Os produtos e as vacas têm seus valores genéticos preditos mais afetados pela heterogeneidade de variâncias do que os touros.

O uso de diferentes critérios de comparação dos resultados é recomendado em estudos de avaliação dos efeitos da heterogeneidade de variância, pois, sob determinados critérios, tais efeitos podem não ser evidenciados.

As avaliações genéticas ponderadas utilizadas neste estudo podem ser feitas de forma a reduzir riscos e são indicadas, porque apresentam resultados iguais ou superiores àqueles obtidos pela avaliação que assume homogeneidade de variância.

\section{Literatura Citada}

BOLDMAN, K.G.; FREEMAN, A.E. Adjustment for heterogeneity of variances by herd production level in dairy cow and sire evaluation. Journal of Dairy Science, v.73, p.503-512, 1990.

BULMER, M.G. The effect of selection on genetic variability. American Naturalist, v.105, p.201, 1971.

DONG, M.C.; MAO, I.L. Heterogeneity of (co)variance and heritability in different levels of intraherd milk production variance and of herd average. Journal of Dairy Science, v.73, p.843-851, 1990.

GARRICK, D.J.; Van VLECK, L.D. Aspects of selection for performance in several environments with heterogeneous variances. Journal of Animal Science, v.65, p.409-421, 1987.

HENDERSON, C.R. Applications of linear models in animal breeding. Ghelph: University of Ghelph, 1984. 462p.
HILL, W.G. On selection among groups with heterogeneous variance. Animal Production, v.39, p.473-477, 1984.

MEUWISSEN, T.H.E., Van Der WERF, J.H.J. Impact of heterogeneous within herd variances on dairy cattle breeding schemes: a simulation study. Livestock Production Science, v.33, p.31-41, 1993.

REVERTER, A.; TIER, B.; JOHNSTON, D. J. et al. Assessing the efficiency of multiplicative mixed model equations to account for heterogeneous variance across herds in carcass scan traits from beef cattle. Journal of Animal Science, v.75, p.1477-1485, 1997.

SAS INSTITUTE Statistical Analysis System User's Guide. Version 6.12. Cary: 1996.

SCHAEFFER, L.R. Linear models and computing strategies in animal breeding. Guelph: University of Guelph, 1993. $168 \mathrm{p}$.

SCHENKEL, F.S.; BRITO, F.V. The GENSYS method for genetic evaluation of beef cattle. In: WORLD CONGRESS ON GENETICS APPLIED TO LIVESTOCK PRODUCTION, 5., 1994, Guelph. Proceedings... Guelph, 1994. v.17, p.192-195.

TORRES, R.A. Efeito da heterogeneidade de variância na avaliação genética de bovinos da raça holandesa no Brasil. Belo Horizonte: Universidade Federal de Minas Gerais, 1998. 124p. Tese (Doutorado em Ciência Animal) Universidade Federal de Minas Gerais, 1998.

VINSON, W.E. Potential bias in genetic evaluations from differences in variation within herds. Journal of Dairy Science, v.70, p.2450-2455, 1987.
Recebido em: 15/10/01

Aceito em: 10/04/02 